\title{
Marco Regulatorio para la InClusión de Estudiantes con Necesidades Educativas Especiales en Chile: un Análisis en Torno a Prácticas ESCOLARES $^{1}$ \\ Normative FRAMEWORK FOR THE INCLUSION OF STUDENTS WITH SPECIAL EDUCATIONAL NEEDS IN CHILE: AN ANALYSIS OF SCHOOL PRACTICES
}

\author{
Pablo SANDOVAL ${ }^{2}$ \\ Rosario PALACIOS 3 \\ Sofia LARRAZABAL ${ }^{4}$ \\ Rocío BERWART ${ }^{5}$
}

\begin{abstract}
RESUMEN: Presentamos una revisión del estado de la legislación sobre educación especial en Chile y un análisis cualitativo de su aplicación en escuelas regulares con Programas de Integración Escolar. Exponemos que la normativa para la integración de niñas/ os con Necesidades Educativas Especiales se enmarca en un contexto enfocado en la rendición de cuentas sin criterios comunes que faciliten su interpretación. Como consecuencia, los actores involucrados ponen en práctica las normas de diversas formas, lo que incluye el abordar la normativa respecto a asuntos administrativos y financieros para intentar que no obstaculice la inclusión de manera universal.
\end{abstract}

PALABRAS CLAVE: Legislación educacional. Inclusión educacional. Administración educativa.

\begin{abstract}
We present a review of the state of legislation on special education in Chile and a qualitative analysis of the concrete application of this regulation in regular schools with School Integration Programs. We expose that the regulations governing the integration of children with Special Educational Needs into regular classes are part of a broader legal context characterized by dispersion and little systematization, focus on accountability and lack of common criteria to facilitate their interpretation. As a result, the actors involved program the standards in a variety of ways, including addressing regulations regarding administrative and financial matters to try not to hinder inclusion in a universal way.
\end{abstract}

KEYWORDS: School legislation. Educational inclusion. Educational administration.

\section{INTRODUCCIÓN}

La educación inclusiva, fuertemente asociada a la educación especial en Chile, representa una perspectiva útil para analizar cómo transformar los entornos de aprendizaje para responder a la diversidad de los estudiantes, la cual implica múltiples categorías como el género, la pobreza, la discapacidad, la etnia y la raza, el idioma, entre otras (United Nations Educational, Scientific and Cultural Organization, [UNESCO], 2005). Un enfoque inclusivo que contemple a "todos sin excepción” también significa abandonar etiquetas estigmatizadoras impuestas a

\footnotetext{
${ }^{1}$ https://doi.org/10.1590/1980-54702021v27e0083

${ }^{2}$ Licenciado en ciencias jurídicas y sociales Universidad de Chile. Abogado socio de SLS \& Abogados. Santiago/Chile. E-mail: psandoval@slsabogados.cl. ORCID: https://orcid.org/0000-0003-3639-3961

${ }^{3}$ Doctora en sociología London School of Economics and Political Science. Investigadora Asociada del Centro de Justicia Educacional. Santiago/Chile. E-mail: r.palacios@uc.cl. ORCID: https://orcid.org/0000-0001-8839-3520

${ }^{4}$ Licenciada en ciencia política y relaciones internacionales Universidad Alberto Hurtado. Asistente de investigación del Centro de Justicia Educacional. Santiago/Chile. E-mail: sofia.larrazabal@gmail.com. ORCID: https://orcid.org/0000-0001-5868-1820

${ }^{5}$ Licenciada en sociología Pontificia Universidad Católica de Chile. Investigadora cualitativa. Santiago/Chile. rocio.berwart@ gmail.com. ORCID: http://orcid.org/0000-0002-9062-0793
} 
niños/as basadas en las categorías de las que son portadores/as (UNESCO, 2020). Al respecto, la política de inclusión educativa en Chile se ha abordado desde el paradigma de regulación de la educación especial para estudiantes con discapacidad y/o Necesidades Educativas Especiales [NEE] (López et al., 2014; Manghi et al., 2020), intentando superar la integración (desarrollo del aprendizaje en espacios compartidos pero poco participativos en relación a las capacidades de los/as niños/as con NEE), y avanzar hacia la inclusión (los espacios y la forma de enseñanza se adaptan para que todos/as puedan participar y aprender) (Infante, 2010; Molina, 2015; Rubio, 2017; Sinisi, 2010).

La evolución jurídico-administrativa y del discurso público sobre la inclusión en Chile ha sido registrada en estudios previos, donde encontramos la centralidad que ha tenido el concepto de inclusión en las últimas reformas legales al sistema escolar. Estas están abocadas a la eliminación de la segregación socioeconómica, a la atención de la diversidad de perfiles estudiantiles existente y a la incorporación de estudiantes excluidos/as por discapacidades o NEE a las escuelas regulares (García \& López, 2019; Infante \& Matus, 2009; Manghi et al., 2020; Treviño et al., 2014).

Dado lo anterior anticipamos una ambivalencia entre el espíritu de la norma chilena y la práctica pedagógica en las salas de clase. Notamos que nominalmente se persigue una noción de cobertura y acceso a la educación coherente con la obligatoriedad constitucional de respetar y promover derechos humanos consagrados en tratados internacionales. Por ejemplo, en la Declaración Universal de Derechos Humanos (1948), el Pacto Internacional de Derechos Económicos, Sociales y Culturales (1966), la "Declaración de Salamanca” (1994) y la Declaración de Derechos de las Personas con Discapacidad (2008), se resaltan la igualdad, la diversidad y el derecho a la educación. No obstante, la política pública que se implementa en las escuelas fue diseñada dentro de paradigmas político-educativos de Nueva Gerencia Pública [NGP] que promueven la estandarización del desempeño de docentes y estudiantes a través de baterías de evaluación de conocimientos curriculares, formularios administrativos, sistemas web de rendición de cuentas y evaluaciones de desempeño docente para medir y jerarquizar la calidad educativa (Assaél et al., 2014; Duk \& Murillo, 2019; Fardella et al., 2016; Manzi et al., 2014; Molina, 2015; Sisto, 2019; Villalobos \& Quaresma, 2015), los que finalmente se transforman en un obstáculo para la implementación de prácticas pedagógicas inclusivas ya que son herramientas rígidas que no ofrecen espacio para la diversidad de situaciones.

Nuestra hipótesis se centra en que un desarrollo normativo desarticulado da paso a tensiones entre las leyes y las prácticas docentes y administrativas dentro de las escuelas que acogen a estudiantes con NEE. Por una parte, las escuelas se ven obligadas a competir por recursos y a postergar orientaciones pedagógicas para las NEE que no tienen cabida en el esquema de la NGP, y por otra, profesores y estudiantes intentan a pesar de ello lograr la inclusión dentro de la sala de clases.

Tras detallar nuestra metodología, el artículo se divide en dos partes: primero presentamos un barrido teórico-normativo para dar cuenta del estado de la legislación en materia de educación especial y NEE. Luego, a través de un análisis de prácticas escolares abordamos las NEE como una dimensión de la inclusión escolar, relevando las principales tensiones entre el contenido de la regulación y su puesta en práctica. 


\section{Metodología}

Para la revisión descriptiva de la normativa sobre educación especial se ordenó el corpus legal vigente en una pirámide kelseniana, entendida como la ordenación jerárquica del sistema de normas que tiene por cúspide la Constitución Política de la República, de la que se desprenden escalonadamente leyes interrelacionadas de mayor a menor rango (Kelsen, 2009). Revisamos la Ley General de Educación (2009), la Ley de Subvenciones y sus modificaciones (1998 en adelante), las Normas sobre Igualdad de Oportunidades e Inclusión Social de Personas con Discapacidad (2010) y la Ley de Inclusión Escolar (2015). Finalmente, se revisaron normas reglamentarias para la inclusión de discapacidades y NEE: los decretos $n^{\circ}$ 170 (2010) y no 83 (2015). Como fuente de consulta se utilizaron las versiones oficiales de las normas, descargadas de las páginas web de sus entidades de origen

Los relatos de prácticas docentes pertenecen a un estudio cualitativo mayor que tuvo como objetivo describir y analizar las políticas públicas orientadas a la inclusión educativa de estudiantes con NEE. Para él se seleccionaron seis establecimientos educacionales en Santiago de Chile, tres adscritos al sistema de administración pública y tres al semi-público (particulares subvencionados). Todos ellos receptores de subvenciones estatales entre las que se cuenta la subvención por estudiante perteneciente al Programa de Integración Escolar [PIE]. En términos de distribución geográfica, cinco escuelas pertenecen a la Región Metropolitana y una a la Región de O’Higgins.

Los hallazgos provienen de la sistematización abierta, axial y selectiva (Flores \& Naranjo 2014; Strauss \& Corbin, 2002; Taylor \& Bogdan, 2000) de datos obtenidos en observaciones no participantes en 18 lecciones co-docentes de Matemáticas y 18 de Lenguaje, en tres niveles escolares (1ero Básico, 4to Básico y Enseñanza Media). También se observaron y analizaron las reuniones de planificación de dichas asignaturas a las que fuimos invitadas como investigadoras. Tanto las/os directivas/os como los planteles docentes fueron informados de nuestra presencia en las aulas, permitiendo su participación de modo voluntario. El registro de las observaciones se realizó mediante redacción de notas de campo. En paralelo se realizaron entrevistas individuales semi-estructuradas con profesores/as especiales, intérpretes de lengua de seńas y personal directivo, y luego grupos de discusión con duplas docentes -y sus intérpretes, según fuese el caso-. Estos métodos fueron aplicados previo consentimiento de las/os participantes y tanto las entrevistas como los grupos fueron grabados y transcritos. Todos los fragmentos que se presentan se encuentran anonimizados.

El análisis conjunto y relacionado de los hallazgos del barrido normativo y de los datos cualitativos relevó ejes jurídicos, políticos y socioculturales (Hodder, 2000), entre los que se encuadran las prácticas de la ley que observamos dentro de las aulas regulares con estudiantes con NEE.

\section{RESULTADOS: INCLUSIÓN Y EDUCACIÓN ESPECIAL EN LA REGULACIÓN JURÍDICA CHILENA}

La regulación es un campo de estudio interdisciplinario (Levi-Faur, 2010) enfocado en el rol estatal para delimitar actividades mediante leyes, oficios, decretos, circulares, etc., constituyéndose como una de las diversas formas de ejercer el poder en las sociedades modernas (Black, 2002). Precisamente en función de ello, la cuestión no es si la educación se regula o no, 
sino cómo y en qué extensión se regula (Levin \& Belfield, 2003). Aunque en Chile existe nutrida literatura sobre la regulación constitucional del derecho a la educación y sobre la libertad de enseñanza (Flores, 2014; Nogueira, 2008; Salazar, 2020; Vivanco, 2007, entre otros), no existe todavía un estudio sistemático del universo de normas que regulan la educación chilena, salvo quizás por el trabajo de Zárate (2021).

Actualmente, la Ley General de Educación (Ley n² 20.370) es el cuerpo normativo más importante del sistema educacional chileno. Define la educación como un proceso de aprendizaje permanente que abarca distintas etapas de la vida de las personas y cuya finalidad es alcanzar el desarrollo espiritual, ético, moral, afectivo, intelectual, artístico y físico, mediante la transmisión y el cultivo de valores, conocimientos y destrezas (Art. 2 Ley n 20.370, 2009). La educación se manifiesta a través de la enseñanza regular, estructurada por niveles y modalidades que aseguran la unidad del proceso educativo y facilitan la continuidad del mismo a lo largo de la vida de las personas (Art. 2 Ley n² 20.370, 2009). Las modalidades educativas se definirán como opciones organizativas y curriculares de la educación regular para dar respuesta a requerimientos específicos de aprendizaje, personales o contextuales, con el propósito de garantizar la igualdad en el derecho a la educación (Art. 22 Ley n² 20.370, 2009). Así, la educación especial definida en el artículo 23 de la LGE constituye una modalidad específica del sistema educacional chileno que desarrolla su acción de manera transversal en los distintos niveles, tanto en los establecimientos de educación regular como especial, proveyendo un conjunto de servicios, recursos humanos, técnicos, conocimientos especializados y ayudas para atender las necesidades educativas especiales que puedan presentar algunos alumnos de manera temporal o permanente a lo largo de su escolaridad, como consecuencia de un déficit o una dificultad específica de aprendizaje (Art. 23 Ley n $\left.^{\circ} 20.370,2009\right)$.

Esta definición está estrechamente relacionada con aquello que la ley llamará "Necesidades Educativas Especiales": un alumno presenta NEE cuando precisa ayudas y recursos adicionales, ya sean humanos, materiales o pedagógicos, para conducir su proceso de desarrollo y aprendizaje (Art. 23, Ley n² 20.370, 2009). Queda en evidencia, que tanto la definición legal de educación especial como la definición de NEE se centran en las prestaciones pertinentes (servicios, recursos humanos, técnicos, conocimientos especializados y ayudas) para el acceso educativo de una persona diagnosticada por un/a profesional de la salud.

Es importante distinguir entre los conceptos de NEE y discapacidad. La normativa chilena define "persona con discapacidad" como aquella que teniendo una o más deficiencias físicas, mentales o sensoriales, de carácter temporal o permanente, ve impedida o restringida su participación plena y efectiva en la sociedad en igualdad de condiciones con las demás (Art. 5, Ley no 20.422, 2010). Al referirse a las medidas que el Estado y las instituciones sociales deben implementar para la igualdad de oportunidades en materia educativa, la norma señala claramente que las NEE podrán estar asociadas o no a una discapacidad (Art. 35, Ley no 20.422, 2010). Dicho de otro modo, para la legislación educacional chilena el requisito habilitante para recibir educación especial no es necesariamente tener una discapacidad, sino requerir un apoyo para el aprendizaje, apuntando hacia la diversidad de condiciones que se puede presentar en una sala de clases. En esta línea, la Ley de Inclusión (Ley n $\left.{ }^{\circ} 20.845,2015\right)$ señala que es deber del Estado "promover que se generen las condiciones necesarias para el acceso y permanencia de los estudiantes con NEE en establecimientos de educación regular o especial” (Art. 2a, Ley n ${ }^{\circ}$ 
20.845, 2015). Destacamos también la incorporación de un principio de integración, apelando a la inclusión, cuyo objetivo es "eliminar todas las formas de discriminación arbitraria que impidan el aprendizaje y la participación de los y las estudiantes” (Art. 1k, Ley n² 20.845, 2015).

Considerando la segregación en la educación producto de la mercantilización introducida desde políticas y programas de NGP (Queupil \& Durán del Fierro, 2018), la Ley de Inclusión introduce mecanismos compensatorios como la modificación de la selección de la matrícula en establecimientos escolares y el desarrollo curricular de diversos perfiles de estudiante. A su vez la LGE prohíbe que se condicione la incorporación, asistencia y permanencia de estudiantes al consumo de medicamentos para tratar trastornos de conducta, instando a los establecimientos a propiciar iniciativas de apoyo biopsicosociales y espacios educativos heterogéneos. Ergo, la noción de inclusión que se promueve en la regulación nacional pone énfasis en la integración social para conformar espacios educativos heterogéneos, apelando al acceso a la educación sin discriminaciones y pensando en las aulas como espacios inclusivos donde conviven estudiantes con diferente capital cultural, económico y social (Queupil \& Durán del Fierro, 2018). No obstante, notamos que la conceptualización de la inclusión en los documentos de la década actual aún corresponde a la de los documentos internacionales de los 90, y se instala en una educación pública invadida por la NGP. Además, la implementación de la inclusión se plantea a través de decretos compensatorios a la integración que no cambian el modelo de base y continúan operando bajo una visión médica que etiqueta -y con ello segrega- a los estudiantes con NEE o discapacidad (López et al., 2018).

Así, en el cotidiano de las escuelas, el Decreto Ley no 170 de 2009 del Ministerio de Educación [MINEDUC] es esencial pues norma los criterios de salud para realizar diagnósticos que fundamenten la subvención estatal por NEE transitorias y permanentes; recomienda reglas sobre la periodicidad y el procedimiento de los diagnósticos y determina el perfil de los profesionales competentes que deberán realizarlos. Finalmente, regula el contenido sustantivo del Programa de Integración Escolar [PIE] en su diseño, requisitos para su aprobación ministerial, mínimo de horas semanales de apoyo profesional y máximo de alumnos con NEE por curso. Por su parte, el Decreto no 83 del 2015 del MINEDUC define orientaciones de diversificación universal del aprendizaje curricular para la educación, para que así los establecimientos educacionales, con o sin PIE, puedan planificar propuestas educativas pertinentes y de calidad para los/as estudiantes que lo requieran. En su Anexo encontramos principios que orientan la adecuación curricular, una conceptualización de NEE, una estrategia general de respuesta a la diversidad denominada "Diseño Universal para el Aprendizaje" [DUA], y la descripción de diversos tipos de adecuaciones curriculares (de acceso y de objetivos de aprendizaje), además de normas específicas para el proceso de toma de decisiones en la implementación de adecuaciones curriculares.

Las definiciones presentadas hasta ahora resultan al menos indiciarias de una comprensión asistencialista del Estado hacia la educación, limitado al aspecto prestacional de acceso. La implementación de este tipo de lógicas produce efectos inesperados en las escuelas donde se implementan respuestas educativas a las demandas de inclusión: Dominique Manghi et al. (2020) son certeras al señalar estos efectos en las culturas escolares, aún funcionales a la clasificación de estudiantes de acuerdo a sus características, ritmos, estilos, comportamiento, etc., siempre en contraste con los parámetros de normalidad: 
Es común escuchar hablar del estudiante "especial", "diferencial”, "integrado", connotando en esa clasificación de otredad; dada la competencia que predomina dentro de las aulas en un sistema que busca alcanzar las metas de aprendizaje en vez de formar ciudadanos para la sociedad, en ocasiones los estudiantes no pueden alcanzar dichas metas en el mismo plazo que el resto de los compañeros [...] se levantan aparatajes técnicos como papeleos, capacitaciones para diagnosticar y obtener subvención estatal para niños con etiquetas que permiten que llegue más dinero a las escuelas, haciendo surgir, por ejemplo, no solo personas "anormales" sino también personas encargadas de etiquetarlos. (Manghi et al., 2020, p. 131-132)

\section{Discusión de hallazgos: de las TENSIONES ENTRE POlíticas Y PRÁCTiCAS PARA La INCLUSIÓN EN ESCUELAS, HACIA LAS TÁCTICAS PARA ENFRENTARLAS}

La normativa descrita se presenta dentro de las escuelas articulada en un conjunto de documentos, acciones, decisiones y prácticas que pretenden solucionar o prevenir una situación dentro de un espacio contextualizado, sobre el cual la autoridad genera circunstancias para reducir el rango de opciones que tienen los actores ante una problemática determinada (Ball et al., 2012; Braun et al., 2010; Singh et al., 2014; Velásquez, 2009). Así, la normativa educacional cumple con aquella asentada idea de que la regulación implica siempre una intervención intencional en las actividades de una población determinada (Koop \& Lodge, 2015). Los aprendizajes y el desarrollo integral de los estudiantes no solo dependen de sus NEE, sino también de las realidades cotidianas que surgen en cada escuela: organización escolar, estrategias de aprendizaje, esfuerzos y metas docentes, interacciones con la familia e intereses de niñas/ os en sus propios procesos educativos, entre otros (Molina, 2015).

La implementación de las regulaciones dependerá de múltiples personas, necesidades, recursos, etc. usualmente desconocidos por quienes diseñan las políticas que se implementan dentro de las aulas (Braun et al., 2010). Por ello, un enfoque etnográfico en educación especial permite registrar y comprender de mejor modo que la diada integración/inclusión es más compleja que el análisis puntual de la otredad de niños/as integrados/as en escuelas regulares (Sinisi, 2010), pues "permite relevar la complejidad de la trama de sentidos de los sujetos que participan de ese proceso - docentes, niños, familias, especialistas, etc.- disputando los significados sobre lo que se entiende que puede ser la experiencia escolar inclusiva” (Sinisi, 2010, p. 13).

\subsection{INCLUSIÓN MÁS ALLÁ DE LA NORMA}

Si bien existe un "derecho a la educación especial", al ser ésta una modalidad educativa, es optativa para las escuelas. Esta opción exige el diseño de un PIE como requisito para recibir una subvención, limitando la cantidad de estudiantes con NEE que puede haber dentro de las aulas -un máximo de 2 alumnos con NEE permanentes y 5 con NEE transitorias. Muchos estudiantes con NEE, especialmente aquellas no diagnosticadas, quedan formalmente fuera del PIE. Esto no impide que existan intentos por resistir la restricción numérica como criterio para la inclusión en la práctica:

El foco tiene que estar puesto en la estrategia para todos, con o sin necesidades educativas, porque aún cuando el Decreto te indica que son cinco estudiantes transitorios y dos permanentes, eso no significa que otro estudiante no tenga necesidades educativas. Es solamente un tema administrativo, no tiene que ver con la práctica educativa en sí, es para regular los recursos del programa para la contratación de profesionales. (Nelson, psicólogo PIE escuela México, Región de O’Higgins). 
Aún cuando los establecimientos acepten que la inclusión dentro del aula debe ser para todos/as los/as estudiantes, pertenezcan o no al PIE, y las/os estudiantes que no están inscritos por falta de cupos reciban especial atención dentro de la sala de clases, éstos quedan sin ser reconocidos jurídicamente como portadores de un derecho a una atención especializada fuera del aula y de un seguimiento sistemático de sus aprendizajes en el marco de su NEE.

\subsection{Modelo de financiamiento y Nueva Gerencia Pública}

El artículo $9^{\circ}$ del Decreto nº 2 (1998) del MINEDUC sobre subvenciones del Estado a establecimientos educacionales crea dos subvenciones específicas para la educación especial: "Educación Especial Diferencial" y "NEE de Carácter Transitorio"6 para financiar establecimientos que impartan educación especial y a establecimientos regulares que cuenten con un PIE. El monto total recibido por concepto de subvención escolar, cuando se trate de alumnos con NEE en escuelas regulares, podrá fraccionarse y pagarse en relación a horas de atención que requieran. El financiamiento tiene como requisito el diseño e implementación de un PIE con cupos asociados a un diagnóstico médico, lo que en la práctica, cuando en una escuela no se llenan todos los cupos (principalmente a consecuencia de la necesidad de diagnósticos médicos específicos y normados), constrińe los recursos para contratar docentes, paradocentes y profesionales asociados (terapeutas ocupacionales, fonoaudiólogos, psicólogos, etc.) en cantidades suficientes para cubrir la demanda de NEE. La escasez de recursos se observa especialmente en establecimientos pequeños, en los cuales el número de estudiantes pertenecientes al PIE es también menor:

Belén: logramos que la escuela contratara a otra fonoaudióloga que vea a los niños que quedan fuera del PIE. Entonces, ella toma a todos estos niños con trastornos transitorios, con algunas dislalias, y algunos TEA también...

Laura: estamos muy cargadas a un curso por nivel [porque no hay recursos para contratar profesoras diferenciales para los dos cursos], entonces hay una diversidad que en realidad aterra y que de repente escapa de nuestras manos atender; por mientras, el otro curso, teniendo igual hartas necesidades de apoyo, queda abandonado.

Ana: cuando trabajamos con los cuartos básicos "integrados" hay una presión mayor por el tema SIMCE7. Y de verdad que es una gran presión. Este es un colegio con excelentes resultados, por ańos hemos mantenido la excelencia, hay puntajes altos y nosotros no podemos fallar. (Grupo de Discusión Escuela Argentina, Región Metropolitana).

Notamos que al no poder contar con recursos para pagar a una profesora especial para cada curso en el nivel nivel, algunas escuelas optan por juntar a todos los/as estudiantes

\footnotetext{
${ }^{6}$ Se entenderá por "Necesidades Educativas Especiales de Carácter Transitorio" a "aquellas (necesidades) no permanentes que requieran los alumnos en algún momento de su vida escolar a consecuencia de un trastorno o discapacidad diagnosticada por un profesional competente, y que necesitan de ayudas y apoyos extraordinarios para acceder o progresar en el currículum por un determinado período de su escolarización”. Esto incluye condiciones como el déficit atencional y trastornos específicos del lenguaje y aprendizaje.

${ }^{7}$ Sistema de Medición de la Calidad de la Educación: batería de pruebas de Lenguaje y Matemáticas, Ciencias Naturales y Sociales, Historia y Geografía, e Inglés, además de recoger información sobre docentes, estudiantes, padres y apoderados a través de cuestionarios anexos. Sus resultados otorgan puntajes a los colegios según niveles de logro y así estos son ordenados según su desempeńo Los establecimientos que reiteradamente obtienen bajos puntajes pueden ser intervenidos por el Estado e incluso pueden ser cerrados.
} 
con NEE en una misma sala, lo que dificulta la labor de las docentes y también segrega a los estudiantes según sus características. Se dan casos en que un cuarto básico, por ejemplo, es el cuarto básico "PIE" (con estudiantes con NEE o discapacidad) y el otro es el "bueno" (en el que es fácil enseñar porque no hay estudiantes con NEE o discapacidad) ya que este nivel rinde las pruebas SIMCE, por lo que etiqueta y segrega estudiantes, y se presiona a las docentes a conseguir resultados que aseguren un buen puntaje para el establecimiento.

\subsection{RENDICIÓN DE CUENTAS Y TRABAJO COLABORATIVO}

El trabajo de las profesoras de educación especial se ve especialmente influido por la perspectiva gerencial de la regulación, pues la necesidad de acreditar permanente los diagnósticos de sus estudiantes mediante el llenado de un set de formularios en plataformas limita su presencia enseñando en aula:

Marcelo: (Profesor Básico Ed. Física): Hay una evaluación que se nos ha pedido, que no sé cuántas hojas son... 14 hojas. Yo tengo ocho niñas, entonces imagínese todo lo que estuve ahí. A lo mejor que buena sería esta información si realmente se hiciera una retroalimentación con ella, pero yo creo que eso no se hace. Perdón que yo sea así, pero se gasta tiempo, hojas y harta información en llenarlo, ¿̇y para qué me dedico a llenarlo super detallado? si es para tener una estadística...

Florencia (Educadora Diferencial): pero nosotras tenemos 40 y tantas niñas por cada una, entonces imagínate tantos papeles por niña, suman y suman páginas. (Grupo de Discusión Escuela Holanda, Región Metropolitana).

A pesar de las particularidades propias que supone la atención de niñas/os con NEE, las educadoras diferenciales - al no contar con un régimen laboral específico - tampoco cuentan con más horas no lectivas para destinar no sólo a estas labores de rendición de cuentas, sino que a tareas propias de su profesión tales como la creación de material didáctico, el diseño universal de contenidos, las adecuaciones curriculares y la atención personalizada a estudiantes, lo que muchas veces realizan durante horas lectivas, las cuales legalmente solo deberían destinar a docencia de aula.

\subsection{TENSIONES Laborales Y PROFESIONALES EN EL MODELO NORMATIVO}

El relato anterior también revela roces en términos de prioridades para los/as profesores/as en relación a la inclusión y a los aspectos administrativos que ella exige. Esto permite que la cultura escolar se configure en torno a una constante auditoría que difumina los roles pedagógico-didácticos de quienes trabajan en los PIE:

En el transcurso de las políticas públicas y los cambios de gobierno, las nuevas innovaciones que hay en tendencia educativa empezaron a poner en jaque, o en relieve, la palabra "inclusión" como un tema mayor, que abarca no solamente las NEE sino que de forma más general, a todo el curso, siendo capaz de atender toda la diversidad que hay en las salas de clases. Entonces, en esa tarea, a nosotros los educadores diferenciales se nos cambia un poco el rol, se nos cambian las formas de trabajo y también, tenemos que cambiar nuestra estructura y función en relación a lo que veníamos haciendo, pero nadie nos dijo nada, no se consultó, siguen impartiendo las carreras de pedagogía con el modelo antiguo, cuando tú llegas a trabajar ya no es el modelo con el que a ti te educaron y te enseñaron. Por lo tanto ahí comienza a haber como una especie, para 
mí, de nebulosa en cuál es nuestra función real, con lo que significa ahora la inclusión. (Grupo de Discusión Escuela Perú, Región Metropolitana).

Compartir el aula con otro/a docente ha detonado tensiones entre profesionales, limitando las posibilidades de una enseñanza inclusiva. La co-docencia requiere coordinación para confeccionar y registrar planificaciones curriculares, lo que no siempre ocurre, aun cuando las horas de planificación estén consideradas en el horario de los profesores regulares y les sean debidamente remuneradas, cosa que no siempre ocurre para los/las profesores diferenciales:

Yo tengo el horario súper establecido, pero los profesores [regulares] se me pierden o piden esa hora para salir al supermercado. Entonces, la orden para las profes del PIE es que si yo me quedé de juntar para planificar a la $1 \mathrm{PM}$, y a la una el colega no está, yo registro y me voy, porque yo no tengo por qué estar buscando a mi colega por cielo, mar y tierra. ¿Entonces qué pasa? La próxima semana la profe del PIE no tiene idea lo que van a hacer porque no hubo planificación [...] Lo que yo hago, básicamente, es "acusar" ante la directora, quien entonces hace una sanción, pero al final esa hora de planificación se les paga igual, pero a nosotras [educadoras diferenciales] no nos pagan esa hora de planificación. (Entrevista Coordinadora PIE Escuela Suiza, Región Metropolitana).

Cabe señalar que la mención docente de educación especial no es equivalente en términos administrativos a las menciones de los docentes regulares en asignaturas como lenguaje o matemáticas, lo que implica que las horas de planificación que sí son pagadas para docentes regulares, no lo son en el caso de las y los educadores especiales. A esto se suma que, aunque existan sanciones por la inasistencia de docentes regulares a las planificaciones, en lo concreto estas prácticas no tienen efecto porque no contemplan la recuperación de la hora de planificación perdida, con todas las consecuencias que aquello trae a la hora de realizar la labor conjunta en el aula.

\section{Conclusiones}

Como ha sido fundamentado, recae sobre el Estado garantizar la inclusión educativa, y con ello la obligación de proveer y financiar la modalidad de educación especial. Sin la preocupación por la inclusión educativa no es posible comprender adecuadamente la garantía del derecho a la educación para todas/os. Sin embargo, la praxis de esta obligación presenta diversas complejidades que se desprenden del propio marco regulatorio debido a que este reduce su comprensión de inclusión a prestaciones de acceso a la educación y a la rendición de cuentas.

Podemos concluir que la regulación sobre educación especial se encuentra fuertemente anclada a la obtención de subvenciones estatales con diversos requisitos de rendición de cuentas, los cuales más que promover el paradigma de la inclusión, establecen obligaciones que sostenedores/as y profesoras/es debe cumplir para obtener recursos. La tensión entre la obligatoriedad y la opcionalidad es una muestra significativa de la prevalencia de un modelo de integración, que se supera solo en casos donde docentes pueden ejercer un alto grado de agencia para ir más allá del sentido prestacional e incluir a sus estudiantes de modo integral en el aprendizaje de contenidos curriculares y habilidades personales. 
El modelo de atención por cupos asignados mediante un diagnóstico médico es una medida resistida por las comunidades mediante la adaptación de la norma para hacer frente a realidades pedagógicas que exceden lo contemplado por quienes han diseñado regulaciones educativas.

El financiamiento individual asociado a una NEE atenta directamente contra el principio mismo de la inclusión, pues los recursos están destinados a una población diferencial y no a un programa para todos/as. A nivel de contratación de docentes, paradocentes y profesionales asociados, se dificulta la capacidad de los equipos PIE de dar abasto a las demandas de NEE en las aulas. Así, no es posible garantizar la inclusión educativa dentro de un marco legal que regula un sistema basado en la competencia por recursos y en la rendición de cuentas como eje central.

Las culturas escolares no fueron preparadas para la inclusión y por ende no se orientan hacia ella, generando tensiones laborales y profesionales en las prioridades que cada docente debe cumplir. La gestión administrativa de los PIE no hace más que intensificar estos conflictos y obliga a docentes diferenciales a abandonar horas de aula, de planificación y de creación de material educativo para completar formularios de evaluación y constancias sobre el trabajo realizado.

Si bien es sabido que la cultura no se implementa normativamente, en este caso las normas dificultan el desarrollo de una cultura inclusiva. La escuela brinda oportunidades de desarrollo y aprendizaje para la diversidad, y por ello la normativa que las rige debiese apuntar a implementar estructuras que faciliten una cultura inclusiva para dar respuestas educativas significativas a todas/os las/os estudiantes, y atender a sus particularidades y cualidades, sin distinciones ni adaptaciones curriculares forzadas, proponiendo un currículo común donde implícitamente se realicen adaptaciones para los alumnos y alumnas con NEE (Molina, 2015). Esto a su vez responde a la visión inclusiva de las políticas educativas en una era globalizada, que implica unificar y potenciar el trabajo de los actores educativos para generar políticas equitativas de calidad. Desde esta perspectiva, la educación regular y la educación especial no deberían ser consideradas como realidades separadas, sino que, por el contrario, tendrían que entenderse como una unidad que trabaja colaborativamente para lograr los principios de una educación para todos/as (Danforth \& Naraian, 2015; Molina 2015; Naraian, 2021).

A la luz estos hallazgos, especialmente considerando las experiencias de la aplicación de la normativa en las escuelas, creemos prioritario revisar y modificar la regulación sobre educación especial. En particular, nos parece central revisitar los paradigmas de enseńanza imperantes para evitar que la rendición de cuentas difumine roles didáctico-pedagógicos y limite la diversificación y planificación curricular, entre otras consecuencias.

\section{REFERENCIAS}

Assaél, J., Acuña, F., Contreras, P., \& Corbalán, F. (2014). Transformaciones en la cultura escolar en el marco de la implementación de políticas de accountability en Chile. Un estudio etnográfico en dos escuelas clasificadas en recuperación. Estudios Psicológicos, XL(2), 7-26. https://doi.org/10.4067/ S0718-07052014000300001_

Ball, S., Maguire, M., \& Braun, A. (2012). How schools do policy: policy enactments in secondary schools. Routledge. https://doi.org/10.4324/9780203153185 
Black, J. (2002). Regulatory conversations. Journal of law and society, 29(1), 163-196. https://doi. org/10.1111/1467-6478.00215

Braun, A., Maguire, M., \& Ball, S. (2010). Policy enactments in the UK secondary school: Examining policy, practice and school positioning. Journal of Education Policy, 25(4), 547-560. https://doi. org/10.1080/02680931003698544

Danforth, S., \& Naraian, S. (2015). This new field of inclusive education. Beginning a dialogue on conceptual foundations. Intellectual and Developmental Disabilities, 53(1), 70-85. https://doi. org/10.1352/1934-9556-53.1.70

Decreto con fuerza de ley no 2, de 28 de noviembre de 1998. MINEDUC (Sobre subvención del Estado a establecimientos educacionales). https://www.leychile.cl/Navegar?idNorma=127911\#sostenedor0

Decreto Exento $n^{\circ}$ 83, de 05 de febrero de 2015. MINEDUC (Aprueba criterios y orientaciones de adecuación curricular para estudiantes con necesidades educativas especiales de educación parvularia y educación básica). https://www.bcn.cl/leychile/navegar?idNorma=1074511

Decreto $n^{\circ}$ 170, de 21 de abril de 2010. MINEDUC (Fija normas para determinar los alumnos con necesidades educativas especiales que serán beneficiarios de las subvenciones para educación especial). https://www.leychile.cl/Navegar?idNorma=1012570\&idVersion=2010-08-25

Duk, C., \& Murillo, J. (2019) Editorial: Segregación escolar y meritocracia. Revista Latinoamericana de Educación Inclusiva, 13(1), 11-13. https://doi.org/10.4067/S0718-73782019000100011

Fardella, C., Sisto, V., Morales, K., Rivera, G., \& Soto, R. (2016). Identidades laborales y ética del trabajo público en tiempos de rendición de cuentas. Psykhe, 25(2), 1-11. https://doi.org/10.7764/ psykhe.25.2.789

Flores, J. C. (2014). Derecho a la educación: su contenido esencial en el derecho chileno. Estudios Constitucionales, 12(2), 109-136. http://doi.org/10.4067/S0718-52002014000200005

Flores, R., \& Naranjo, C. (2014). Análisis de datos cualitativos: el caso de grounded theory-Teoría Fundamentada. En M. Canales (Ed.), Escucha de la escucha: análisis e interpretación en la investigación cualitativa (pp. 75-114). LOM.

García, R., \& López, V. (2019). Políticas de Educación Especial en Chile (2005-2015): continuidades y cambios. Revista Brasileira de Educação Especial, 25(1), 1-16. https://doi.org/10.1590/s141365382519000100001

Hodder, I. (2000). The interpretation of documents and material culture. En N. Denzin, \& Y. Lincoln (Eds.), Handbook of qualitative research (pp. 393-402). Sage.

Infante, M. (2010). Desafíos a la Formación Docente: Inclusión Educativa, Estudios Pedagógicos, 36(1), 287-297. https://doi.org/10.4067/S0718-07052010000100016

Infante, M., \& Matus, C. (2009). Policies and practices on diversity: Reimagining possibilities for new discourses, Disability and Society, 24(4), 437-445. https://doi.org/10.1080/09687590902879049

Kelsen, H. (2009). Teoría pura del derecho. Eudeba.

Koop, C., \& Lodge, M. (2015). What is regulation? An interdisciplinary concept analysis. Regulation and Governance, 11(1), 95-108. https://doi.org/10.1111/rego.12094

Levi-Faur, D. (2010). Regulation and regulatory governance. Jerusalem Papers in Regulation and Governance. Working Paper no 1 (47 páginas). 
Levin, H., \& Belfield, C. (2003). The Marketplace in Education. Review of Research in Education, 27, 183-219. https://doi.org/10.3102/0091732X027001183

Ley $n^{o}$ 20.370, 2 de julio de 2010. General de Educación. https://www.bcn.cl/leychile/ navegar?idNorma $=1006043$

Ley $n^{\circ}$ 20.422, 10 de febrero de 2010. Establece normas sobre igualdad de oportunidades e inclusión social de personas con discapacidad. https://www.bcn.cl/leychile/navegar?idLey=20422

Ley $n^{\circ} 20.845,8$ de junio de 2015. De inclusión escolar que regula la admisión de los y las estudiantes, elimina el financiamiento compartido y prohíbe el lucro en establecimientos educacionales que reciben aportes del Estado. https://www.leychile.cl/Navegar?idNorma=1078172

López, V., Julio, C., Morales, M., Rojas, C., \& Pérez, M. V. (2014). Barreras culturales para la inclusión: políticas y prácticas de integración en Chile. Revista de Educación, 363, 256-281.

López, V., González, P., Manghi, D., Ascorra, P., Oyanedel, J. C., Redón, S., Leal, F., \& Salgado, M. (2018). Políticas de inclusión educativa en Chile: tres nudos críticos. Archivos Analíticos de Políticas Educativas, 26(157), 96-129. https://doi.org/10.14507/epaa.26.3088

Manghi, D., Conejeros, M. L., Bustos, A., Aranda, I., Vega, V., \& Diaz, K. (2020). Comprender la educación inclusiva chilena: panorama de políticas e investigación educativa. Cadernos de Pesquisa, 50(175), 114-135. https://doi.org/10.1590/198053146605

Manzi, J., Bogolasky, F., Gutiérrez, G., \& Grau, V. (2014). Análisis sobre valoraciones, comprensión y uso del SIMCE por parte de directores escolares de establecimientos subvencionados. https://politicaspublicas. uc.cl/wp-content//uploads/2016/04/Informe-Final-F711269-Manzi.pdf

Molina, Y. (2015). Necesidades educativas especiales, elementos para una propuesta de inclusión educativa a través de la investigación acción participativa: el caso de la Escuela México. Estudios pedagógicos (Valdivia), 41(especial), 147-167. https:// doi.org/10.4067/S0718-07052015000300010

Naraian, S. (2021). What can "inclusion" mean in the post-human erra? Journal of disability studies in education, 1, 1-21. https://doi.org/10.1163/25888803-bja10001

Nogueira, H. (2008). El derecho a la educación y sus regulaciones básicas en el derecho constitucional chileno e internacional de los derechos humanos. Ius et Praxis, 14(2), 209-269. https://doi. org/10.4067/S0718-00122008000200007

Queupil, J. P., \& Durán del Fierro, F. (2018). El principio de inclusión: similitudes y diferencias en la educación escolar y superior en Chile. Revista Latinoamericana de Educación Inclusiva, 12(1), 111 128. https://doi.org/10.4067/S0718-73782018000100111

Rubio, V. (2017). Inclusión de personas en situación de discapacidad en educación superior, desde el enfoque de la responsabilidad social, en un contexto de transiciones discursivas respecto del binomio integración/inclusión. Revista Latinoamericana de Educación Inclusiva, 11(2), 199-216. https://doi.org/10.4067/S0718-73782017000200013

Salazar, S. (2020). Jurisprudencia constitucional sobre los derechos educacionales Requisitos minimos, limites y regulación. Editorial Tirant lo Blanch.

Singh, P., Heimans, S., \& Glasswell, K. (2014). Policy enactment, context and performativity: ontological politics and researching Australian national partnership policies. Journal of education policy, 29(6), 826-844. https://doi.org/10.1080/02680939.2014.891763 
Sinisi, L. (2010). Integración o inclusión escolar: ¿un cambio de paradigma? Boletín de Antropología y Educación, 01, 11-14.

Sisto, V. (2019). Inclusión "a la Chilena": La inclusión escolar en un contexto de políticas neoliberales avanzadas. Archivos Analiticos de Politicas Educativas, 27(23). https://doi.org/10.14507/ epaa.27.3044

Strauss, A., \& Corbin, J. (2002). Bases de la investigación cualitativa. Técnicas y procedimientos para desarrollar la teoría fundamentada. Editorial Universidad de Antioquía.

Taylor, S., \& Bogdan, R. (2000). Introducción a los métodos cualitativos de investigación. La búsqueda de significados. Paidós.

Treviño, E., Valenzuela, J. P., \& Villalobos, C. (2014). ¿Se agrupa o segrega al interior de los establecimientos escolares chilenos? Universidad de Chile.

United Nations Educational, Scientific and Cultural Organization. (2005). Orientaciones para la Inclusión: Asegurar el Acceso a la Educación para Todos. UNESCO.

United Nations Educational, Scientific and Cultural Organization. (2020). Inclusión y Educación: todos y todas sin excepción. Informe de seguimiento de la educación en el mundo. UNESCO.

Velásquez, R. (2009). Hacia una nueva definición del concepto "política pública”. Desafíos, 20, 149-187.

Villalobos, C., \& Quaresma, M. L. (2015). Sistema escolar chileno: características y consecuencias de un modelo orientado al mercado. Convergencia, 22(69), 63-84. https://doi.org/10.29101/crcs. v22i69.3634

Vivanco, Á. (2007). Derecho a la educación y libertad de enseñanza: un aparente conflicto y sus efectos sobre una proposición normativa en Chile. Dirección de Asuntos Públicos, Facultad de Derecho UC, 2(8), 1-19.

Zárate, M. (2021). La educación como actividad económica. Bases para una crítica al sistema educacional chileno. Ediciones DER.

Recebido em: 21/01/2021

Reformulado em: 01/06/2021

Aprovado em: 20/07/2021 
SANDOVAL, P. et al.. 